\title{
Tratamiento del Tumor de Wilms
}

\author{
Dra. Paola Zolezzi R. ${ }^{1,2}$; Dra Maria Soledad Wenzel A. ' \\ Dra. Cecilia Faúndez V.; Dra. Bernardita Cardemil J.; Dr. Luis Norambuena $\mathrm{M}^{\mathbf{4}}$. \\ Dr. Roberto Horsella R. ${ }^{5}$; E.L. Marion Hertmann H. $^{6}$
}

\section{Wilms' Tumor: Results of Treatment}

From january 1981 to december 1986,6 nale patients with Wilms' tumor were seen at the regional hospital of Valdivia, at southern Chile. Five of them had "favorable" histology. One patient was classified in stage I, two in stage II and three in stage Ill. The treatnient regimen consisted in nephrectomy at the affected side and chemotheraphy for 15 months, on the basis of two drugs (actinomycin $D$ and vincristine) for stage I. In stages II and III chemotheraphy was done with three drugs (actinomycin D, vincristine and adriamycin). Radiotherapy was added to stage II (3.000 cgy to the area of the tumor) and III $\{2.000 \mathrm{gy}$, total abdominal irradiation and $1.500 \mathrm{cgy}$ to the tumoral area). Treatment was well tolerated in all cases and the 3 -year relapse-free survival sate was $75 \%$ for the whole group.

(Key words: Wilms' tumor, treatment, arvival).

Ell tumor de Wilms (TW) se presenta con una frecuencia anual aproximada de 7,8 casos por un milón en EE.UU. en menores de 15 años, siendo el tumor maligno más común del tracto genitourinario en el niño ${ }^{1}$.

Modificaciones sucesivas en el tratamiento asociado de cirugia, radioterapia y quimioterapia de combinación realizadas en forma colaborativa a partir de 1969 , han elevado notablemente la sobrevida en el TW ${ }^{2}$. Resul. tados publicados en $1981^{\mathbf{3}}$ establecen para pacientes de grupo I, con tumor confinado al rinón, completamente extirpado, no sometidos d radioterapia, tratados con actinomicina D más vincristina, ya sea durante 6 meses o 15 meses, una sobrevida libre de recidiva de $88 \%$ a dos años plazo, Para los pacientes en Grupos II y llI, con lesiones tumorales locales más avanzadas y para los de Grupo IV con metástasis a distancia, tratados todos con radio-

1. Instituto de Pediatría, Lniversidad Austral de Chile, Valdivia.

2. Instituto de Hematología, Universidad Austal de Chile, Valdivia.

3. Instituto de Especialidades, Universidad Austral de Chile, Valdivia.

4. Instituto de Patologia, Universidad Austral de Chile, Valdivia.

5. Servicio de Radiología, Hospital Regional de Vat divia.

6. Servicio de Pediatría, Hospital Regional de Valdjvia. terapia posterior a la cinugia y actinomicina D más vincristina, la subrevida libre de recidiva a dos años plazo es de $63 \%$ y para los que recibieron ademas adriamicina como tercera droga, la sobrevida libre de recidiva a dos años plazo aumenta a $77 \%$.

E] resultado de la aplicación de pautas basadas en protocolos extranjeros ${ }^{2}, 3$ y nacionales ${ }^{4}$ en un número reducido de pacientes con $T W$ es cl motivo de esta comunicación.

\section{MATERIAL Y METODO}

Entre enero de 1981 y diciembre de 1984 ingresaron al servicio te pediatría del Hospital Regional de Valdivia 6 niños con TW. El estudio clínico previo a la cirugía se basó en nomas impartidas por GOPECH ${ }^{4}$. Para la cirugía se siguieron las recomendaciones de Leape y col. ${ }^{5}$ y de Martín y col. ${ }^{16}$. La clasifícación en el grupo cousespondiente a cada paciente, dependiendo ésta del grado de extensión anatómica de la enfermedad, se hizo según D'Angio y $\mathrm{col}^{3}$. Para la diferenciación del TW en subtipos histopatólógicos se siguió el criterio establecido por Beckwith y Palmer?

La quimioterapia de combinación, basada en las proposiciones de Jenkin ${ }^{8}$, consistió para los pacientes del grupo $\mathrm{I}$ en actinomicina D (AMI): 0,015 mg $\mathrm{x} \mathrm{kg} \mathrm{x}$ día e.v., una dosis diaria por 5 días consecutivos en las semanas $1 ; 6 ; 12 ; 25 ; 38 ; 51$ y 64 y vincristina (VCR): $1,5 \mathrm{mg} x \mathrm{~m}^{2} \mathrm{x}$ dosis e.v., una dosis semanal durante las primeras 8 semanas, $y$ continuando con irual dosis en el dia 1 y 8 de las siguientes curas con AMD. En los niños con tumores de los grupos II y III se agregó adriamicina (ADR); $60 \mathrm{mg} x \mathrm{~m}^{2}$ e.v. por una vez cada tres meses, comenzando 6 semanas después de la cirugía. En estos grupos no se efectuó la cura de la semana 6 con AMD. 
La radioterapin (RDT) empleada se basó en Cassady y col. ${ }^{9}$, adminjstrándose en el grupo $113.000 \mathrm{cgy}$ al lecho tumoral(fosa renal) y en el grupo III $2.000 \mathrm{cgy}$ de irradiación abdominal total y 1.000 a $1.500 \mathrm{cy}$ de irradiación adicional al lecho tumorat.

Durante el seguiniento los pacientes fueron controlados con examen físico, hemograma completo antes, durante $y$ después de cada cura de quimioterapia $y$ durante el período de radioterapia, perfil bioquimico $y$ radiografía de tórax cada tres meses, pielografía de eliminación a los seis meses y al finalizar el tratamiento. Posteriormente los pacientes se controlaron cada cuatro meses con hemograma completo, perfil bioquimico, examen de orina y radiografía de tórax.

La sobrevida actuarial $x$ analizó de acuerdo al método de tablas de vida 10 .

\section{RESULTADOS}

Los seis pacientes son de sexo masculino y menores de 4 años, siendo uno de ellos lactantes mayores en el momento del diagnóstico. El motivo de hospitalización fue en todos una masa abdominal, agregảndose hernaturia macroscópica en dos.

Después de la nefrectomia del rinón afectado y de realizar el estudio histologico. un paciente fue clasificado en grupo I; 2 en grupo Il y 3 en grupo III.

En la clasificación en subtipos histopatologicos cinco pacientes tenian histologra favo. rable: uno de predominio epitelial dos de predo. minjo blastematoso y dos de subtipo mixto. En el caso con histología desfavorable esta corres. pondía a un subtipo mixto con anaplisia difusa.

La quimioterapia planificada se realizó completa en cinco casos. Uno de los riños del grupo III no acudió a control después de la tercera cura con AMD más VCR, encontrándose libre de recidiva demostrable en la exploración de uso habitual.

La radioterapia planificada para los casos en los grupos II y III se realizó en forma completa.

Durante la asociación de radioterapia $y$ quimioterapia se observaron infecciones moderadas: cuatro pacientes sufrieron neumopatías agudas comprobadas radiológicamente, uno diarrca aguda con coprocultivo positivo para pseudomona aeruginosa; dos niños sufrieron varicela y en uno hubo pigmentación folicular de predominio abdominal. Durante la fase del tratamiento con quimioterapia exclusiva ocurieron infecciones intercurrentes de fácil resolución, presentando varicela un tercer pacientc. En ambas fases se observaron vómitos de intensidad variable coincidiendo con el uso de AMD y granulocitopenias de rápida recuperación.

En los controles realizados una vez finalizado el tratamiento no se han observado hasta ahora recidivas tumorales.
Uno de los niños ingresó 17 ñeseses después de finalizado su tratamiento a raíz de síntomas abdominales agudos secundarios a obstrucción intestinal por vólvulo, practicándose en la operación destorción de éste, seguido 48 horas después por resección intestinal y yeyunostonía. Durante el postoperatorio evolucionó mal, presentando nueva obstrucción intestinal por el cual fue reintervenido efectuándose enterolisis, enteroanastomósis término-terminal yeyuno-yeyuno, yeyuno-ileon, yeyunostomía e ileostomia. En las sucesivas intervenciones no se encontró evidencia de tumor residual, siendo los informes histológicos negativos así como el estudio clínico, radiológico y de laboratorios practicado. Pese al tratamiento con antibióticos realizado y a la nutrición parenteral, falleció 37 días después de su ingreso, nо autorizando los padres la necropsia.

No se han observado hasta ahora secuelas atribuibles a radioterapia o quimioterapia, habiendo ingresado el primer paciente hace 66 meses y el último hace 27 meses.

En diciembre de 1986, 4 pacientes estaban vivos libres de recidiva. I desaparecido del control en remisión completa y 1 fallecido sin recidiva tumoral.

En este grupo, la sobrevida actuarial a 36 meses ha sido de $75 \%$.

\section{DISCUSION}

Sí bien el númcro de pacientes de esta muestra es escaso, la sobrevida libre de recidiva en tres años de $75 \%$ es un logro aceptable. ya que $91 \%$ de las recidivas en el TW se presentan en los 18 meses siguientes a la nefrectomía' '. En una revisión reciente de la literatura de la lengua inglesa ${ }^{12}$, se han encontrado 13 casos de TW recidivados después de un intervalo libre de enfermedad de 5 años, constituyendo un factor de riesgo la asociación entre nefroblastomatosis y $\mathbf{T W}^{13}$. Entre las características de nuestro grupo que favorecerían los resultados obtenidos está, probablemente, el claro predominio dc subtjpos histopatológicos favorables, ya que la sobrevida a dos años es de $90 \%$ para estos casos y del $54 \%$ cuando la histopatología es desfavorable ${ }^{3}$, hecho analizado recientemente en nuestro medio ${ }^{14}$. Sc tuvo especial cuidado en descartar formas que pudiezan cortesponder al tumor rabdoide del riñón de probable origen neurcectodérmico $y$ al sarcoma de variedad de células claras, que fueron incluidos anteriomente como $\mathrm{TW}^{7}$, y que constituyen entidades diferentes de peor pronóstico ${ }^{15}$. Otro factor favorable es la falta de compromiso de los linfáticos abdominales, ya que se han 
registrado $54 \%$ de sobrevida en los pacientes con invasión de los linfáticos regionales y $82 \%$ cuando no hay compromiso ganglionar ${ }^{3}$.

La ausencia de metástasis en el momenta de diagnóstico inicial, permite también resultados mejores, si bien se ha comunicado recientemente que en los pacientes del grupo IV con histología tavorable, la mortalidad a dos años plazo ha bajado a $9 \% 16$.

En relación al tratamiento, informes prelimi. nares recientes ${ }^{17}$, basados en las tasas de sobrevida libre de enfermedad a dos años plazo. permiten señalar tos siguientes hoclios:

En los pacientes en grupo 1 con histología favorable se obtienen iguales resultados usando (AMD-VCR), tanto durante 10 semanas como por 6 meses. En los pacientes con tumores grupos II $y$ III, los resultados fueron levemente superiores con el uso de tres drogas (AMD-VCRADR). En el grupo II no hubo diferencias al asociar RDT (2.000 cgy). Para los pacjentes en el grupo IV y los que prestan histología desfavorable, tratados todos con RDT y durante 15 meses con quimioterapia, los resultados fueron mejores con tres drogas (AMD-VCR-ADR) que si se agrega una cuarta droga (ciclofosfamida).

Si bien en nuestro grupo no se han detectado secuelas atribuibles a la quimioterapia o la radioterapia, los pacientes con TW deben controlarse por períodos de tiempos prolongados, ya que resultados preliminares serialan una mayor incidencia de complicaciones musculoesqueléticas, quedando por determinarse cardiovasculares $y$ otras $^{18}$.

\section{RESUMEN}

En el período enero 1981 a diciembre 1986 se registraron en el servicio de Pediatría del Hospital Regional de Valdivia sejs niños con Tumor de Wilms, cinco de eltos con histología favorable, encontrándose un paciente en grupo I, dos en grupo II $y$ tres en grupo III. Los pacientes fueron sometidos a un protocolo consistente en nefrectomía del riñón afectado y quimioterapia durante 15 meses, en base a dos drogas para el grupo 1 (actinomicina D más vincristina) y a tres drogas para los grupos II y III (actinomicina D, vincristina y adriamicina). Se asoció radioterapia en el grupo II de $3.000 \mathrm{cgy}$ al lecho tumoral y en el grupo III de $2.000 \mathrm{cgy}$ de irradiación abdominal total y 1.500 cgy al lecho tumoral. El tratamiento fue bien tolerado en todos los casos y la sobrevida libre de recidiva a tres años plazo es de $75 \%$ para el total del grupo.

\section{REFERENCIAS}

1. Green. DM: The diagnosis and management of Wilms' Tumor. Pcdiatr Clin North Am 32: 735, 1985 .

2. Breslow, N.E., Palmer, N.F., Hill, L.R., Buning, J., D'Angio, G.J.: Wilms' Tumor: Prognostic factors for patients without metastases at diagnosis. Cancet 41: 1577, 1978.

3. D'Angio, G.J., Evans, A., Breslow, N., Beckwith, B., Bishop, H., Farewell. V., Goodwin, W., Leape. L., Palmer, N., Sinks, L., Sutow, W., Tefft. M., Wolff. J.: The treatment of Wilms' Tumor: Results of the Second National Wilms' Tumor Study. Cancer 47: 2302, 1981 .

4. GOPECH: Principalos enfermedades oncológicas infantiles: Normas terapéuticas $y$ de estudio, 1981, Pp $56-60$.

5. Leape, LL., Breslow. N.E., Bishop, H.C.: The sugical treatment of Wims' Tumzor: Results of the National Wilns' Tumor Study, Ann Surg. 187: $351,1978$.

6 Martin, L.W., Schaffner, D.P., Cox J.A., Rosenkrantz, J.G., Richardson, W.R.: Retroperitoneal lymph node dissection for Wilm' Tumor. J Pediatr Surg 14: 704, 1979.

7. Beckwith, J.B., Palmer, N.F.: Histopathology and prognosis of Wilms' Tumor: Results from the First National Wins' Tumor Study. Cancer 41: $1937,1978$.

8. Jenkin, R.D.: The treatment of Wilms' Tumot. PediatI Clin North Am 23: 137-160, 1976.

9. Cossady, J.R., Joffe, N.. Paed, D., Feller. R.: The increasing importance of radiation theraphy in the improved prognosis of children with.Wilms' Tumor. Cancer 39: 825, 1977.

10. Futson J.M. Kent, M., Ekert, H, Waters, K.D. The treatment of Wims' Tumor: Results from Royal Children's Hospital, Melbourne, 1967-1977. J Pediatr Surg 18: 235, 1983.

11. Lemerle, J.. Tournade, M.F., Gerard-Marchant, R., Flamant, R., Sarrazin, D., Flamant, F., Lemerie, M., Jundt, S., Zuker,J.M., Schweiguth O.: Wilms' Tumor: Natural history and prognostic factors. Cancer 37: 2557, 1976.

12. Kim Zaatari, G.S., Baum, E.S., Jaffe, N., Cushing, B., Chard, R.L., Swiren, G.T., Beckwith, J.B.: Recurrence of Wilms' Tumor after apparent cure. I Pediatr 107: 44, 1985.

13. Heideman, R.L., Haase, G.M., Foley, C.L., Wilson, H.L., Bailey, W.C.: Nephroblastomátosis and Wilms' Tumor, Cancer 55: 1446, 1985.

14. Les Heras, J, Benveniste, $S$., Kusz, $P$. Campos, $R$. Fernindez, W., Julio, M.: Tumor de Wilns: Histo patología y pronóstico. Rev Chil Pediatr 57: $339,1986$.

15. Palmer, N.F, Susow, W.: Clinical Aspects of the shabdold tumor of the kidney: A report of the Natjonal Wilms' Tumor Study Group. Med Pediatr Oncol 11: 242, 1983.

16. Breslow, N.E, Churchil, G., Nesmith. B., Thomas, P., Beckwith, J.B., Othersen H.B., D'Angio, G.J." $C$ inicopathologic features and prognosis for Wilms' Tumor patients with metastases at diagnosis. Cancer 58: (in press).

17. D. Angio, G.J.; Evans, A.E., Breslow, N., Beckwith, J.B., Baum, E., DeLorimier, $A$., Farewell, $V$., Fernbach, D., Hrsbovsky, E., Jones, B., Kelalis, P. Othersen, H.B., Tefft, M., Thomes, P.R.,: Results of the Third National Wilms' Tumor 
Stludy: A preliminary report. (Abstr.) Proc. Am. Assoc Cancer Res. 25: $183,1984$.

18. Evans, A.E., Breslow, N., Norkool, P., D'Angio,
G.J.: Complications in long-term survivors of Wilms' Tumor. (Abstr.) Proc Am Assoc Cancer Res 27: $204,1986$. 